\title{
Inner Size Effect in Layered Films with Eutectic Interaction of Components
}

\author{
S.V. Dukarov, S.I. Petrushenko*, V.N. Sukhov, I.G. Churilov, A.L. Samsonik \\ AND O.I. SKRYL \\ V.N. Karazin Kharkiv National University, 4 Svobody Sq., Kharkiv 61022, Ukraine
}

(Received May 31, 2017; in final form January 24, 2018)

\begin{abstract}
The results of the study of melting of $\mathrm{Bi}-\mathrm{Sn}$ and $\mathrm{Pb}-\mathrm{Sn}$ polycrystalline layered film systems with the thickness of 200-400 $\mathrm{nm}$ on a substrate with temperature gradient are given. Multilayer (each layer is of 10-20 nm) and bilayer films (layers are of 100-200 nm) of the same total thickness have been investigated. Broadening of the melting range in all films and lowering of melting point in multilayer samples compared to the bilayer ones have been observed. The observed phenomena are discussed within existing thermodynamic concepts in consideration of interfacial energy of contacting layers of components and energy of grain boundaries in polycrystalline films.
\end{abstract}

DOI: 10.12693/APhysPolA.133.1186

PACS/topics: inner size effect, multilayer films, interfacial energy

\section{Introduction}

The size effect of melting temperature was predicted more than a hundred years ago on the basis of thermodynamic considerations and was experimentally confirmed in the $50 \mathrm{~s}$ of the last century. Since then, for many substances, not only the size dependence of the melting point $[1,2]$ has been studied, but also a change in the temperature of other phase transitions has been revealed, as well as many other size effects of solubility [35], wetting [6], the diffusion coefficient $[7,8]$. Currently, the necessity for further miniaturization and increase of the components efficiency of modern micro- and nanoelectronics leads to the fact that further study of lowdimensional systems is not only of a general scientific, but also of great practical importance.

Thus, some studies offer to use arrays of isolated particles as components of biosensors [9] and photocatalytic generators [10]. The silicon dioxide particles doped with nanoparticles of gold, or semiconductors, have a broad perspective of use in medicine and nanoelectronics, lightemitting diodes, quantum computers and solar cells, biomarkers [11, 12]. The need for a detailed study of size effects, especially concerning thermal stability of film systems $[13,14]$ and the diffusion processes in them, is also due to the extensive use in modern technologies of a variety of functional layers that perform buffer, conductive or protective functions. Therefore, an important issue is to reduce the minimum film thickness at which they acquire the electrical continuity $[15,16]$. The use of surfactants makes it possible to provide conductivity in the films with a thickness of several nanometres. At the same time, it is for objects with such typical size that manifestation of size effects can be expected, as it is observed in practice $[1,2,17]$.

*corresponding author; e-mail: petrushenkokhnu@gmail.com
It should be noted that most of the results related to the known size effects, refer to the individual objects (particles, films) with nanometre typical size, which became the subject of research. Recently, however, the composite materials in which nanoparticles of one material are in contact with the solid matrix of another one, gain more significance. The presence of nanosized inclusions allows to change the properties of the original solid matrix $[16,18]$ to a larger extent and in a controlled way. In $[19,20]$ the use of multilayer films was proposed as a model of the "particle in the matrix" objects. It was found that the phase transitions which occur in nanoscale component, can cause a significant change in electrical resistance of the entire composite sample. This phenomenon [21] was used for the study of supercooling during the crystallization of the liquid phase of fusible component in multilayer films. As a result of these studies, it has been found [20] that due to the particular obtaining conditions, in some composite systems, even for objects whose typical size is $10^{6} \mathrm{~nm}$, it is possible to obtain the values of supercooling which exceed considerably those known for bulk samples.

It is reasonable to assume that the nanoscale nature of inclusions or the structural units which constitute the bulk object may also cause a manifestation of size effects, which in this case will have some influence on the bulk sample as a whole. In [22] it has been found that the polycrystalline films are melted in a certain temperature range. The melting point broadening in nanosystems was also observed in [16], where it was explained by the variety of particle sizes in the sample. A similar effect for composite materials in which particles of In-Ga eutectic alloy have been embedded into the solid nanoporous matrix by the acoustic method, was observed in [23]. Further analysis of a number of works leads to the conclusion that the size effects which occur in composite materials, may differ to a large extent from those that are characteristic of free particles. For example, in $[23,24]$ it is shown that the presence of a solid matrix may lead to the sta- 
bilization of the crystal modifications that are unstable in the bulk state. In Ref. [25] it is found out that unlike free particles [26-28], nanoparticles which are embedded in a solid matrix have the supercooling value that may decrease while they decreasing in size. Many researchers point out to the overheating phenomenon [29, 30], which is usually due to the influence of excess pressure that is caused by the presence of the solid matrix.

Size effects associated with the small size of the constituent elements of a massive object, can significantly affect the performance properties of various composite products. This work is devoted to the study of the melting of binary multilayer film systems, which are a convenient model of nanocomposite structures.

\section{Experimental}

As the study objects there have been selected the films of $\mathrm{Bi}-\mathrm{Sn}$ and $\mathrm{Pb}-\mathrm{Sn}$ contact pairs with a total thickness of the 360 and $200 \mathrm{~nm}$, respectively. Samples are obtained by successive condensation of fusible metals in a vacuum of $10^{-7} \mathrm{~mm} \mathrm{Hg}$ on an extended polished steel substrate of $170 \times 60 \times 4 \mathrm{~mm}^{3}$ size. The sketch of the mutual arrangement of the substrate and evaporators in a vacuum chamber is shown in Fig. 1.

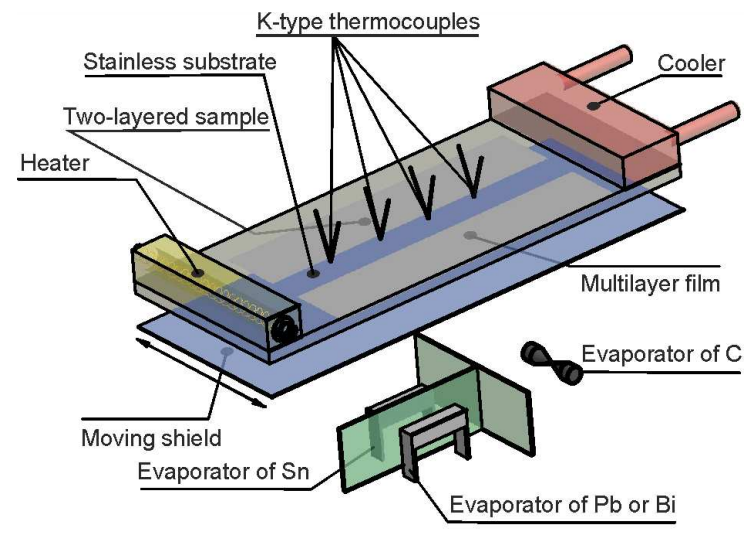

Fig. 1. Sketch of the preparation of two- and multilayer films in a single experiment.

Two series of samples were obtained: multilayer films in which 10 layers of metals alternated between each other and the test two-layer films of the same total thickness. The thickness of the layers in $\mathrm{Bi}-\mathrm{Sn}$ multilayer films was $13 \mathrm{~nm}(\mathrm{Bi})$ and $23 \mathrm{~nm}(\mathrm{Sn})$, and the $\mathrm{Pb}-$ Sn films consisted of layers $10 \mathrm{~nm}$ in thickness. The layers in the test samples were 10 times thicker than in the multilayer films.

$\mathrm{Pb}, \mathrm{Bi}$, and $\mathrm{Sn}$ metals with $99.99 \%$ purity evaporated from separate sources, which were molybdenum $(\mathrm{Pb}, \mathrm{Bi})$ and tantalum (Sn) boats. The metal films were deposited at a rate of $0.5-1 \mathrm{~nm} / \mathrm{s}$ on a substrate at room temperature. The film thicknesses were determined in the course of their preparation by a quartz resonator. Before the condensation of the samples took place an amorphous carbon layer of about $20 \mathrm{~nm}$ thick was deposited on the substrate. Carbon films are poorly wetted by melts of the selected contact pairs that allows to a certain extent to regard the studied films as the free ones. Since the absolute value of the expected inner size effect is assumed to be small, a differential method was used, which consists in the following. In a single vacuum cycle, i.e. under completely identical conditions, on the two halves of a single substrate with the help of a special mobile shield two layered systems were formed: multilayer samples which consist of alternating layers of the components of the studied binary pairs (10 layers of each component), and bilayer films of the same total thickness used as test samples and the behaviour of which should be close to the behaviour of bulk objects.

After condensation completed with the help of the heating unit attached to one edge (heater on the left in Fig. 1) of the substrate and with the forced cooling of the substrate opposite edge (cooler on the right in Fig. 1), temperature gradient was created along it, which is determined according to the data of four K-type thermocouples that are welded to the back side of the substrate. A massive copper block cooled by running water at room temperature was used as a cooler. The measurement error of the sample temperature is about $5 \mathrm{~K}$, but the use of a differential technique allows us to record temperature changes with an accuracy of not worse than $0.1 \mathrm{~K}$. After establishing a stationary temperature gradient films were maintained in the heated state for $20 \mathrm{~min}$, then they were cooled to room temperature, taken from the vacuum chamber and studied with a JEOL JSM-840 scanning electron microscope (SEM).

\section{Results and discussion}

Figure 2 shows photographic images of substrates with $\mathrm{Bi}-\mathrm{Sn}$ and $\mathrm{Pb}-\mathrm{Sn}$ films after their heating in a vacuum chamber on a substrate with a temperature gradient. One can see that, as in other systems that were obtained using similar methods $[6,31]$, in the bilayer and multilayer films two regions of different light scattering are detected. According to many studies [13, 22] the apparent boundary of sharp change of light scattering in the films which are located on the substrate with a temperature gradient, normally corresponds to the melting of the sample. It is proved by the results of electron microscopic studies. Thus, separate particles in the form of a spherical segment are observed in the films above the given boundary (in the temperatures) (Fig. 3b). According to [26-28], metal films melted on a poorly wettable substrate are characterized by such a morphology of the samples. The films remain continuous polycrystalline (Fig. 4) below the boundary, visually observed in Fig. 2. It indicates that they remained solid during heating. If the substrate temperature is lower than the boundary temperature, the film is continuous, and if it is higher the film consists of individual particles of regular spherical shape. The visualization of the boundary between a molten and a continuous film is due to the 
different nature of the light reflection from the film areas with different morphological structure [14]. After melting the film becomes island and diffusively scatters light, while the continuous film reflects light almost specularly.

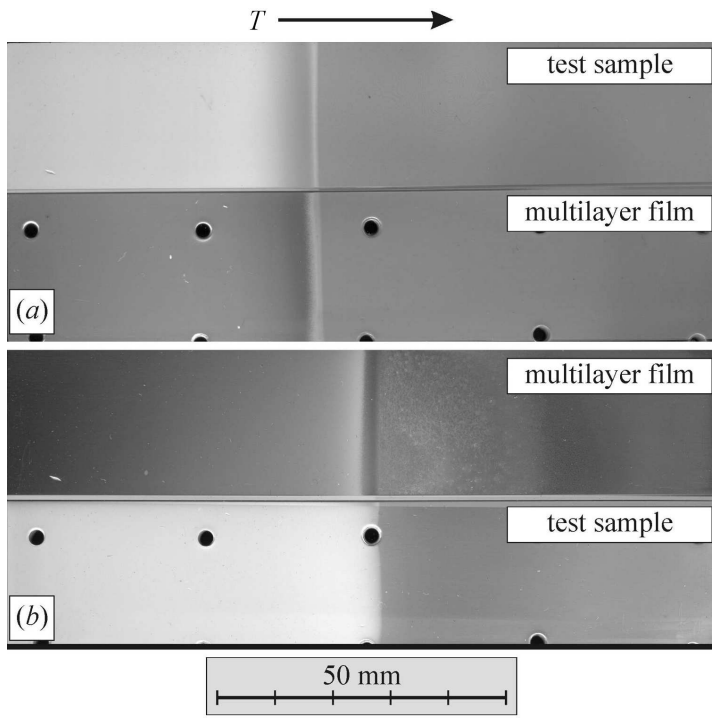

Fig. 2. Photos of the substrates with bi- and multilayer $\mathrm{Bi}-\mathrm{Sn}$ (a) and $\mathrm{Pb}-\mathrm{Sn}$ (b) films.

At the same time it should be noted that the indicated boundaries between the film areas in which they remained crystalline, and those in which their melting occurred, have a finite width, which is especially noticeable in multilayer films. They begin the formation of the transition zone at the temperatures which are lower than in test samples. However, the end temperature of the transition zone is the same for multilayer as well as for bilayer films. Thus its width in multilayer Bi-Sn samples is $2 \mathrm{~K}$ and in $\mathrm{Pb}-\mathrm{Sn}$ is $4.5 \mathrm{~K}$. In test samples the transition zone also has a finite width, which is equal to $0.4 \mathrm{~K}$ for $\mathrm{Pb}-\mathrm{Sn}$ system and in $\mathrm{Bi}-\mathrm{Sn}$ films it is $0.9 \mathrm{~K}$.

According to the electron microscopy studies in the area that separates completely melted and crystalline film areas there are simultaneously solid and liquid phases in multilayer samples during the heating process. The presence of spherical particles whose shape clearly indicates that they were in a liquid state confirms partial melting of the film. The crystalline phase, which has not been melted, is represented in the area by shapeless structures with a flat surface (Fig. 3a). At the same time, bilayer film morphology confirms that they remained in the crystalline state at a temperature corresponding to the transition zone of multilayer films. Along with pores that are typical for thin film samples near the melting point [13], they have crystalline segregations, which, apparently, is an eutectic that is formed by solid-state diffusion (Fig. 4b).

Thus, electron-microscopic studies confirm that the melting of multilayer films with layers of nanoscale thickness takes place in a certain temperature range and starts earlier than in the bulk.

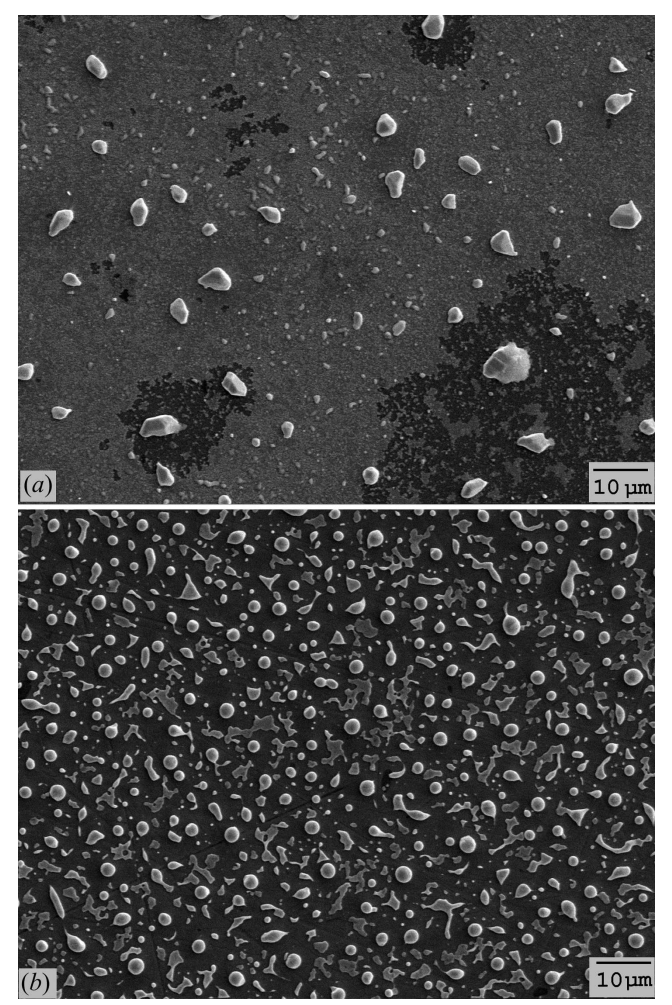

Fig. 3. SEM image of a bilayer (a) and a multilayer (b) $\mathrm{Pb}-\mathrm{Sn}$ film that correspond to heating temperature of $187^{\circ} \mathrm{C}$
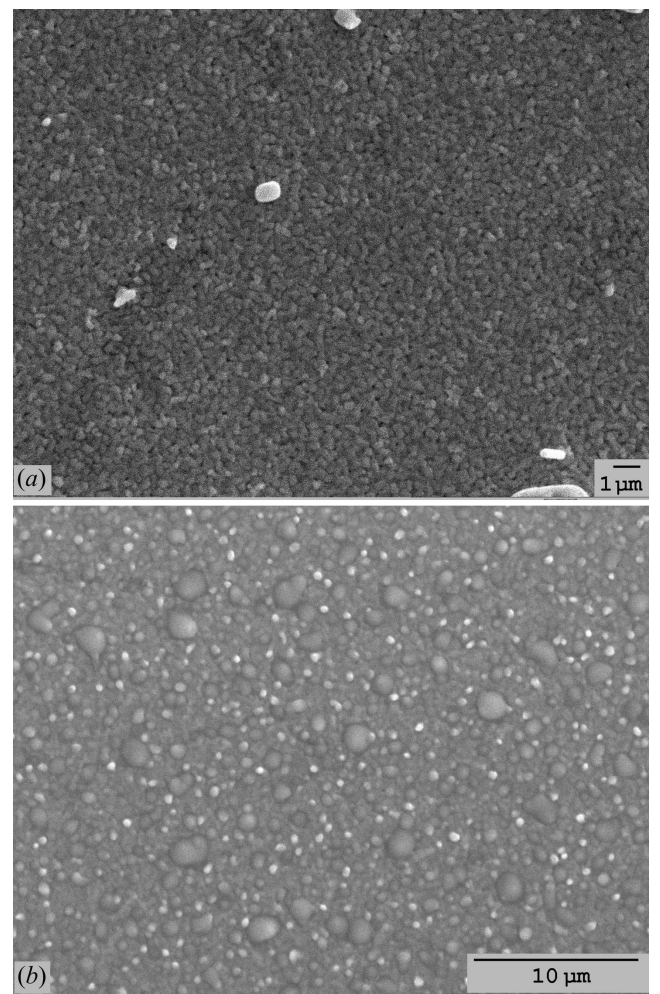

Fig. 4. As in Fig. 3, but for the temperature of $158^{\circ} \mathrm{C}$. 
It should be noted that the size effect of the melting point $[1,2]$, consisting in reducing the phase transition temperature while reducing the film thickness will result in about the same crystal-melt transition boundary shift in bilayer and multilayer films. Therefore, this shift cannot be observed by the differential method. Lowering of the melting point of films of the substances under study with the thickness of more than $100 \mathrm{~nm}$ is less than $0.1 \mathrm{~K}$, that is much smaller than the measurement error of the absolute temperature value provided by a thermocouple.

In the frame of the existing thermodynamic concepts, decrease in onset melting temperature of multilayer films compared to test samples may be due to the presence of additional energy of the interfaces in $\mathrm{Bi}-\mathrm{Sn}$ and $\mathrm{Pb}-\mathrm{Sn}$ films. According to [2] for a multilayer system which is composed of alternating layers of $A$ and $B$ components, for the relative lowering of the melting point $\Delta T / T_{s}$ the following expression can be presented:

$$
\frac{\Delta T}{T_{S}} \approx \frac{\sigma_{A}+\sigma_{B}+\sigma_{A B}(n-1)-2 \sigma_{\ell}}{\frac{n}{2}\left(d_{A}+d_{B}\right) \lambda},
$$

where $\sigma_{A}, \sigma_{B}, \sigma_{A B}$ are the surface energies of free surfaces of $A$ and $B$ components and their boundaries, respectively, and $\sigma_{\ell}$ is the surface energy of the liquid phase based on $A$ and $B$ components taking into account their concentration, $n$ is the total number of layers of $A$ and $B$ components with $d_{A}$ and $d_{B}$, thicknesses, $\lambda$ is heat of fusion per unit volume.

This expression can be presented as

$$
\frac{\Delta T}{T_{S}}=\frac{\sigma_{A}+\sigma_{B}-2 \sigma_{\ell}}{\frac{n}{2}\left(d_{A}+d_{B}\right) \lambda}+\frac{2(1-1 / n) \sigma_{A B}}{\left(d_{A}+d_{B}\right) \lambda} .
$$

Estimates show that when $n$ is large the first term in this expression can be neglected; similarly the value $1 / n$ can be ignored in comparison with unity in the second term. Then to lower the eutectic melting point in the layered film system $\ldots A / B / A / B \ldots$ due to the effect of interfacial energy for the $\mathrm{A} / \mathrm{B}$ interface we obtain

$$
\frac{\Delta T}{T_{S}} \approx \frac{2 \sigma_{A B}}{\left(d_{A}+d_{B}\right) \lambda} .
$$

Thus

$$
\sigma_{A B} \approx \frac{1}{2}\left(d_{A}+d_{B}\right) \lambda \frac{\Delta T}{T_{S}} .
$$

Using the values of the latent heat of fusion [32] from this expression we find out that for the multilayer films the interfacial energy of the interface layers of tin-bismuth is equal to $33 \mathrm{~mJ} \mathrm{~m}^{-2}$, and of those tin-lead is equal to $45 \mathrm{~mJ} \mathrm{~m}{ }^{-2}$. The value of $\sigma$ for $\mathrm{Sn}-\mathrm{Pb}$ films is close to the energy of the solid-liquid boundary given in [33, 34]. Overall, the obtained values of the interfacial energies are of typical values for the contact pairs of fusible metals [35] and are reasonable. It should be noted that the triple point model used to calculate is based on the assumption of form stability during melting. Actually there is a decay of the continuous film, the formation of the array of individual particles and the substrate surface release. These processes are also associated with some changes of the surface energies, that are not taken into account by the model. Therefore, the obtained values should be better regarded as estimated.

Certain broadening of the boundary of crystal-melt transition in bilayer films can be explained as follows. In accordance with the electron microscopy studies (Fig. 4) $\mathrm{Pb}-\mathrm{Sn}$ test samples at a temperature of $158^{\circ} \mathrm{C}$ are polycrystalline and consist of crystallites with a mean size of $300 \mathrm{~nm}$. According to [22] the presence of crystallites of different sizes in the single-component polycrystalline films results in the fact that melting of the sample will occur in a certain temperature range, the value of which is determined by the distribution of crystal grains according to the size and it increases with the reduction of the average grain size.

Probably, this phenomenon could also explain the broadening of the melting range in the studied bilayer films. As the electron microscopy studies show, the mean crystallite area in bilayer $\mathrm{Pb}-\mathrm{Sn}$ films is about $0.1 \mu \mathrm{m}^{2}$, and the length of their boundaries is $1.5 \mu \mathrm{m}$. In accordance with the data of [22] it seems to be sufficient for the manifestation of the inner size effect. However, carrying out of numerical estimates in the studied systems is difficult. The calculation is considerably complicated by the fact that in the multilayer polycrystalline films, there are different types of boundaries (interfacial and grain boundaries), each of which contributes to the broadening of the melting range. Furthermore, boundary identification and determination of their mutual arrangement, in particular for the lower layer, using a SEM is not possible.

It should be noted that in [22] it was found out that the crystallite size in the lead films which were obtained by condensation of substances on the substrate at room temperature must be $10-15 \mu \mathrm{m}$. Significantly smaller size of the crystallites in the studied bilayer films with a component eutectic concentration, probably is due to the formation of eutectic structures in them, which is known to be characterized by the highly dispersed structure. This fact can be used to explain the mechanism of eutectic line imaging in the method of the samples of variable content and variable state [2].

The morphology of the crystalline regions of bilayer and multilayer films, which are close to the melting temperature is significantly different (Fig. 4). Thus, in the multilayer films the surface of many crystallites are rounded, and their area increases by about 15 times compared to the crystallite area of the test films. In this case it is reasonable to assume that immediately after condensing the crystallite size of the multilayer film does not exceed the size of crystallites in test samples. However, during the heating and annealing of samples one should observe the recrystallization which leads to an increase in the crystallite size. If the films are under the same thermal effect, the final crystallite size is determined by the diffusion coefficient that characterizes the system under study. Thus, the increase of the crystallite size in multilayer films, compared with the bilayer films, allows con- 
firming that the average diffusion coefficient in layered film systems with the layer thickness of $10 \mathrm{~nm}$ increases at least an order of magnitude compared with test samples. It should be noted that the increase in the diffusion coefficient in the low-dimensional systems was also observed in other studies [7, 8], and is usually explained by the increasing contribution of the boundaries that usually have a lot of vacancies, which greatly facilitates the diffusion processes at interfaces.

\section{Conclusions}

The presented experimental results show that in both bilayer and multilayer $\mathrm{Bi}-\mathrm{Sn}$ and $\mathrm{Pb}-\mathrm{Sn}$ films with a total thickness of 360 and $200 \mathrm{~nm}$ respectively, a broadening of the melting range is observed, the value of which is significantly greater in multilayer samples than in bilayer samples. In accordance with the current thermodynamic concepts about changing of the nano objects melting temperature, broadening of the melting temperature range in multilayer films is determined by the total thickness of the two contacting thin layers which constitute the layered film system, and of the interfacial energy of their borders. The main contribution to the broadening of the melting border in bilayer samples, is probably due to their polycrystalline structure.

\section{Acknowledgments}

This work was supported by the Ministry of Education and Science of Ukraine.

\section{References}

[1] K. Chopra, Thin Film Device Applications, Springer Science and Business Media, 1986.

[2] N.T. Gladkikh, S.V. Dukarov, O.P. Kryshal, V.I. Larin, V.N. Sukhov, S.I. Bogatyrenko, Surface Phenomena and Phase Transformations in Condensed Films, V.N. Karazin Kharkiv National University, Kharkiv 2004 (in Russian).

[3] A.A. Minenkov, S.I. Bogatyrenko, A.P. Kryshtal, J. Nano-Electron. Phys. 6, 04026 (2014).

[4] S.V. Dukarov, S.I. Petrushenko, V.N. Sukhov, O.I. Skryl, Function. Mater. 23, 218 (2016).

[5] A.P. Kryshtal, S.I. Bogatyrenko, R.V. Sukhov, A.A. Minenkov, Appl. Phys. A 116, 1891 (2014).

[6] S.V. Dukarov, Thin Solid Films 323, 136 (1998).

[7] N.T. Gladkikh, A.P. Kryshtal, S.I. Bogatyrenko, Techn. Phys. 55, 1657 (2010).

[8] A.A. Minenkov, S.I. Bogatyrenko, R.V. Sukhov, A.P. Kryshtal, Phys. Solid State 56, 823 (2014).

[9] H.H. Jeong, A.G. Mark, M. Alarcón-Correa, I. Kim, P. Oswald, T.C. Lee, P. Fischer, Nature Commun. 7, 11331 (2016).

[10] J. Chen, H. Che, K. Huang, C. Liu, W. Shi, Appl. Catal. B Environm. 192, 134 (2016).
[11] V.O. Yukhymchuk, O.M. Hreshchuk, M.Ya. Valakh, M.A. Skoryk, V.S. Efanov, N.A. Matveevskaya, Semicond. Phys. Quantum Electron. Optoelectron. 17, 217 (2014).

[12] N.V. Bondar, M.S. Brodin, N.A. Matveevskaya, Semiconductors 50, 369 (2016).

[13] S.V. Dukarov, S.I. Petrushenko, V.M. Sukhov, R.I. Bihun, Z.V. Stasyuk, D.S. Leonov, Metallofiz. Noveishie Tekhnol. 39, 1069 (2017).

[14] S.I. Petrushenko, S.V. Dukarov, V.N. Sukhov, Vacuum 142, 29 (2017)

[15] R.I. Bihun, O.E. Kravchenko, D.S. Leonov, Ya.A. Pastyrs'kyy, Metallofiz. Noveishie Tekhnol. 35, 603 (2013).

[16] M. Marszalek, A. Polit, V. Tokman, Y. Zabila, I. Protsenko, Surf. Sci. 601, 4454 (2007).

[17] S.I. Protsenko, D.V. Velykodnyi, V.A. Kherai, C.J. Panchal, I.Yu. Protsenko, J. Mater. Sci. 44 , 4905 (2009).

[18] S.S. Chee, J.H. Lee, Thin Solid Films 562, 211 (2014).

[19] S.I. Bogatyrenko, A.V. Voznyi, N.T. Gladkikh, A.P. Kryshtal, Phys. Met. Metallogr. 97, 273 (2004).

[20] S.I. Petrushenko, S.V. Dukarov, V.N. Sukhov, Vacuum 122(A), 208 (2015).

[21] S.I. Petrushenko, S.V. Dukarov, V.N. Sukhov, Probl. At. Sci. Technol. 4, 118 (2016).

[22] S.I. Petrushenko, S.V. Dukarov, V.N. Sukhov, I.G. Churilov, J. Nano- Electron. Phys. 7, 02033-1 (2015).

[23] E.N. Latysheva, A.L. Pirozerskii, E.V. Charnaya, Yu.A. Kumzerov, A.V. Fokin, A.I. Nedbai, A.S. Bugaev, Phys. Solid State 57, 131 (2015).

[24] A.V. Uskov, D.Yu. Nefedov, E.V. Charnaya, J. Haase, D. Michel, Yu.A. Kumzerov, A.V. Fokin, A.S. Bugaev, Nano Lett. 16, 781 (2016).

[25] E. Johnson, H. Henrik Andersen, U. Dahmen, Microsc. Res. Techn. 64, 356 (2004).

[26] N.T. Gladkikh, S.V. Dukarov, V.N. Sukhov, Fiz. Metall. Metalloved. 78, 87 (1994).

[27] N.T. Gladkikh, S.V. Dukarov, A.P. Kryshtal, V.I. Larin, Fiz. Metall. Metalloved. 8551 (1998).

[28] N.T. Gladkikh, S.V. Dukarov, V.N. Sukhov, Zeitschr. Metallk./Mater. Res. Adv. Techn. 87, 233 (1996).

[29] M.J. de Castro, R. Serna, J. Toudert, J.F. Navarro, E. Haro-Poniatowski, Ceram. Int. 41, 8216 (2015).

[30] A. Moros, H. Rösner, G. Wilde, Scr. Mater. 65, 883 (2011).

[31] N.T. Gladkikh, S.V. Dukarov, V.N. Sukhov, I.G. Churilov, Function. Mater. 18, 529 (2011).

[32] C. Morando, O. Fornaro, O. Garbellini, H. Palacio, J. Mater. Sci. Mater. Electron. 26, 9478 (2015).

[33] A.P. Savitskij, L.S. Martsunova, Izv. Tomskogo Politekhnich. Universit. 315, 123 (2009).

[34] M. Gündüz, J.D. Hunt, Acta Metall. 33, 1651 (1985).

[35] Y. Altıntas, E. Öztürk, S. Aksöz, K. Keşlioğlu, N. Maraşlı, J. Therm. Anal. Calorim. 122, 65 (2015). 